\title{
HIGHLIGHTS
}

\section{Challenging the disorders of sex development dogma}

The results of a new investigation cast doubt on the recommendations on gender assignment for a subgroup of 46,XX patients with 21-hydroxylase congenital adrenal hyperplasia (CAH) and Prader 4 or 5 genitalia.

Current guidance suggests raising 46,XX CAH patients who have markedly virilized external genitalia as female. This recommendation is based on the potential for fertility and successful development of female gender identity in a majority of patients. Outcomes data that support this recommendation are limited, however, and anecdotal evidence has indicated that many of the most-severely affected infants actually go on to identify as male.

The results of a survey of 12 such 46,XX CAH patients with Prader 4 or 5 genitalia have been presented in the Journal of Urology. Aged between 35 and 69 years at the time of the survey, the patients had been assigned as male at birth, at a time when newborn screening was not routinely performed.

All but one participant had been diagnosed with 21-hydroxylase CAH during infancy or childhood (at 3-12 years of age). Feminizing surgery had been performed on 2 patients-both subsequently selfreassigned as male. Firm establishment of male identity by the time of diagnosis in the other 9 patients led to an alternate management strategy of total hysterectomy, bilateral salpingoophorectomy, placement of testicular prostheses, and successful repair of hypospadias (in the 5 boys with Prader 4 genitalia). The remaining participant was not diagnosed until adulthood and has always identifed as male.

In adulthood, all reported male gender identity, male gender roles, and sexual orientation to females. Nine have longterm female partners, with 8 reporting strong libido, a functioning penis during intercourse, and frequent satisfactory sexual activity including orgasm.
Lack of familial and social support, and reassignment as female during childhood, were associated with poor self-esteem and body image. A long history of personal problems, including self-harm, suicide attempts, abuse of drugs and alcohol, and lack of career progression, were reported by the 2 patients who had undergone feminizing surgery during childhood.

This study delivers an "eye-opening, mind-twisting, swimming-against-thecurrent message" according to Bradley Kropp, who commented at Faculty of 1000. The article should "open our minds to the fact that what [pediatric urologists] think is dogma today ... may not be the best thing for our patients in the future."

Suzanne J. Farley

Original article Lee, P.A. et al. Should male gender assignment be considered in the markedly virilized patient with 46,XX and congenital adrenal hyperplasia? J. Urol. 184, 1786-1792 (2010) 